\title{
Effect of CKD-MBD therapies on the gastrointestinal expression of phosphate transporters
}

Olivia Carlton; IMPRS ${ }^{1}$; Division of Nephrology ${ }^{2}$; Indiana University School of Medicine ${ }^{3}$, Neal Chen; Division of Nephrology2; Indiana University School of Medicine ${ }^{3}$, Sharon Moe; Division of Nephrology2; Indiana University School of Medicine ${ }^{3}$ Annabel Biruete; Division of Nephrology2; Indiana University School of Medicine ${ }^{3}$

\section{Background}

Chronic kidney disease-mineral and bone disorder (CKD-MBD) is prevalent and encompasses biochemical abnormalities, bone alterations, and vascular calcifications. CKD-MBD treatments include phosphate binders and calcimimetics that effectively lower phosphorus and PTH, respectively, but the impact of these treatments on phosphate transporters in the gastrointestinal tract is still unknown. $\mathrm{NaPi} 2 \mathrm{~B}$ is considered the primary intestinal phosphate transporter. However, NaPi2B inhibition shows limited effectiveness, thus the importance of PIT1 and PIT2 requires further investigation.

\section{Methods}

We tested the effects phosphate binders (ferric citrate $(\mathrm{FC})$ and calcium gluconate $(\mathrm{Ca})$ ) and the calcimimetic KP2326 (KP) in (Cy/+ male rat; CKD) and untreated normal littermates (NL). Treatments lasted 10 weeks until euthanasia at 28 weeks (moderate-to-advanced CKD), where we collected mucosa samples from duodenum, jejunum, and ileum. Blood was collected for biochemistry measurements. Total RNA was isolated, and qPCR performed to assess phosphate transporters expression (NaPi2B, PIT1, PIT2) normalized to b-actin. Results were analyzed via 2-way ANOVA.

\section{Results}

As expected, CKD had abnormal plasma concentrations of phosphorus, PTH, and FGF23. FC and KP effectively lowered phosphorus. KP and Ca lowered PTH, but Ca increased FGF23. $\mathrm{NaPi}$ B $\mathrm{B}$ was expressed in the duodenum and jejunum but not in the ileum, and its expression was upregulated with FC compared to NL. PIT1 was expressed in all segments, but the expression was the highest in the ileum, while PIT2 was constitutively expressed in all segments. Ca led to higher PIT1 and PIT2 expression in the duodenum and jejunum compared to NL, CKD, or KP. KP led to higher expression of PIT1 in the ileum compared to CKD, FC, and Ca.

\section{Conclusions}

CKD-MBD therapies differentially impacted biochemistries and phosphate transporters expression. The effect of $\mathrm{Ca}$ on gastrointestinal expression of PIT1 and PIT2 may explain the higher plasma phosphorus and should be further explored. 